\title{
Examining Manufacturing Readiness for Breakthrough Drug Development
}

\author{
Earl Dye, ${ }^{1,13}$ Annie Sturgess, ${ }^{2}$ Gargi Maheshwari, ${ }^{3}$ Kimberly May, ${ }^{4}$ Colleen Ruegger, ${ }^{5}$ Usha Ramesh, ${ }^{6}$ Heow Tan, ${ }^{7}$ \\ Keith Cockerill, ${ }^{8}$ John Groskoph, ${ }^{9}$ Emanuela Lacana, ${ }^{10}$ Sau Lee, ${ }^{11}$ and Sarah Pope Miksinski ${ }^{12}$
}

Received 15 October 2015; accepted 13 November 2015; published online 25 November 2015

KEY WORDS: breakthrough therapy; CMC; FDA.

\section{INTRODUCTION}

In July 2012, Congress passed the Advancing Breakthrough Therapies for Patients Act as part of the Food and Drug Administration Safety and Innovation Act (FDASIA). Section 902 of FDASIA provides for designation of a drug as a breakthrough therapy "if the drug is intended alone or in combination with one or more other drugs, to treat

The opinions expressed in this manuscript are those of Earl Dye, Annie Sturgess, Gargi Maheshwari, Kimberly May, Colleen Ruegger, Usha Ramesh, Heow Tan, Keith Cockerill, John Groskoph, Emanuela Lacana, Sau Lee, and Sarah Pope Miksinski and do not necessarily reflect the views or policies of the FDA.

${ }^{1}$ Technical Regulatory Policy, Genentech, a Member of the Roche Group, 1399 New York Ave, NW, Suite 450, Washington, DC, Washington 20005, USA.

${ }^{2}$ Regulatory-CMC, Bristol-Myers Squibb Company, New York, New York, USA.

${ }^{3}$ Biologics Process Development \& Commercialization, Merck \& Co., Inc., Kenilworth, New Jersey, USA.

${ }^{4}$ Biologics CMC Regulatory Affairs, Merck \& Co., Inc., Kenilworth, New Jersey, USA.

${ }^{5}$ Technical Research and Development, Novartis Pharmaceuticals Corporation, Hannover, New Jersey, USA.

${ }^{6}$ Regulatory Affairs, CMC, Pharmacyclics Inc., Sunnyvale, California, USA.

${ }^{7}$ Quality and Technical Operations, Pharmacyclics Inc., Sunnyvale, California, USA.

${ }^{8}$ Regulatory Affairs, Amgen, Thousand Oaks, California, USA.

${ }^{9}$ Global Chemistry Manufacturing \& Controls, Pfizer Inc., New York, New York, USA.

${ }^{10}$ Biosimilars and Biologics Policy, Office of Biotechnology Products, CDER, U.S. FDA, 10903 New Hampshire Ave, Silver Spring, 20993, Maryland, USA.

${ }^{11}$ Office of Pharmaceutical Quality, CDER, U.S. FDA, 10903 New Hampshire Ave, Silver Spring, 20993, Maryland, USA.

${ }^{12}$ CDER, U.S. FDA, 10903 New Hampshire Ave, Silver Spring, 20993, Maryland, USA.

${ }^{13}$ To whom correspondence should be addressed. (e-mail: dye.earl@gene.com) serious or life-threatening diseases or conditions and preliminary clinical evidence indicates that the drug may demonstrate substantial improvement over existing therapies (1)." Breakthrough designation is a mechanism that the U.S. Food and Drug Administration (FDA) can grant to sponsors to expedite the development of these promising therapies.

As part of the program, the FDA and sponsor collaborate in a dynamic, multi-disciplinary, resource-intensive process to determine the most efficient path using an "all hands on deck approach" involving senior managers and experienced review staff and more frequent and interactive communications $(2,3)$. The objective is to expedite design and review of the clinical development program so that trials are as efficient as possible, and the number of patients exposed to potentially less efficacious treatment is minimized. As a consequence, clinical development timelines involving the traditional three distinct phases could be reduced from 7-10 to 3-5 years.

The shorter clinical development programs will have significant impact on product and process development timelines requiring the manufacturing organization to reconsider traditional approaches to product and process development and undertake their own resource-intensive, cross-functional team approach to ensure a sustained supply of safe and efficacious product at the time of approval. To ensure success, the manufacturing organization should have good communications with the clinical organization to facilitate identification of potential candidates for breakthrough designation early and help gate or accelerate the appropriate Chemistry, Manufacturing, and Controls (CMC) and current Good Manufacturing Practice (cGMP) development activities. It is important to understand that breakthrough drug development programs are resource intensive; sponsors need to be selective about which programs to take forward and ensure management support. Moreover, a collaborative, cross-functional approach between development, commercial, and regulatory operations, with early and robust discussions, is essential to ensure successful development and launch of a breakthrough drug product.

In March of 2015, Friends of Cancer Research (Friends) convened a group of industry and FDA stakeholders familiar with developing breakthrough drugs to explore options, 
manufacturers of small molecule and biologic products have for front-loading certain critical manufacturing activities to speed development of breakthrough therapy drugs. This expert group also explored options for science- and risk-based approaches to mitigating the potential risk of having less $\mathrm{CMC}$ information at the time of launch versus the benefit of having these innovative new products available to patients sooner. The considerations captured in this white paper outline approaches that sponsors have taken to successfully manufacture breakthrough products as well as new approaches that aim to further explore potential efficiencies in bringing breakthrough products to market. These ideas were presented at a public forum, convened by Friends, on June 10 in Washington, DC, in an effort to seek broad feedback on the recommendations put forth to expedite rate-limiting steps in CMC and cGMP for products demonstrating high clinical benefits while ensuring an adequate supply of safe and efficacious product at the time of approval (4).

\section{BREAKTHROUGH DEVELOPMENT PROGRAMS MAY PUT CMC/GMP ACTIVITIES ON CRITICAL PATH}

Timelines for completing CMC/GMP activities for a breakthrough product will be driven by the design of the clinical development program for the breakthrough product. Each development program will vary depending on the complexity of the product, how soon accelerated CMC development activities begin, availability of platform technology, relevant prior knowledge, and timing of designation. If the breakthrough designation is granted at an early development stage following promising preliminary clinical data, some of the phase III CMC-enabling activities may need to be accelerated. On the other hand, if a breakthrough designation is granted to a product in late stage development, the challenges for manufacturing readiness may be less burdensome but may also need to be addressed in a more compressed time frame. While drugs approved under the breakthrough pathway still need to meet statutory requirement for product quality, safety, and efficacy, balancing risk to product quality and availability for patients is critical. Therefore, development of breakthrough drugs necessitates risk-based approaches to product and process development, commercial readiness, and regulatory filings, with a focus on a reliable supply of quality product available to meet and sustain market demand. To this end, conventional timing for certain activities may be shifted, with some activities starting sooner, some completing later, and others potentially deferring post-filing (e.g., some aspects of process optimization). Additional activities (e.g., increased testing) may also be warranted based on the overall risk of the breakthrough product coupled with available supporting data.

\section{MANUFACTURING CONSIDERATIONS FOR BREAKTHROUGH DRUG DEVELOPMENT}

Some critical product and process characterization activities could be addressed earlier and may facilitate manufacturing readiness for breakthrough products. While the considerations below may aid in introducing efficiencies into the development process, they are not intended to be prescriptive, rather reflective of best practices based on prior experiences or discussions, and rely on establishing early and robust communications with the FDA to ensure suitability with the specific development program. Where appropriate, molecule-specific recommendations are noted for consideration.

\section{In General}

- Selection of the best molecular candidate for development based on physical-chemical properties and the pharmacokinetic (PK) profile for small molecule drugs or screening for and engineering out, where possible, hot spots for degradation or undesired modifications for biologic drugs

- Ensuring the fit of candidate molecules into the manufacturer's platform for drug substance (DS) and drug product (DP) and related processes to improve speed and robustness

- Front-loading activities to address non-platform behavior and/or unusual product and process characteristic

- Assessing Critical Quality Attributes (CQAs) earlier and front-loading method validation activities for them

- Incorporating preliminary quality target product profile (QTPP) and bridging in the development of clinical service dosage forms for early clinical studies (i.e., phase I), which may generate data to support a breakthrough designation (e.g., identification of whether enabling formulations are needed to support rapid development)

Biologics

- Use of cell line and vector constructs for which significant prior knowledge/platform knowledge is available (e.g., viral safety aspects), with the clone selected for phase I studies, ideally carrying through to commercialization, thus minimizing any comparability concerns arising from cell line changes; appropriate methods should be used to establish clonality

- Assuring preliminary cell line stability for launch should be demonstrated (e.g., limit of in vitro cell age validation)

- Design and use of host cell protein assays that are comprehensive in their coverage and can be used for multiple products (from the early stages of development and all the way through commercialization)

- Performing sequence variant analysis early in development and on aged cells to understand and control potential cell line variability

\section{Small Molecules}

- Early identification of the most thermodynamically stable salt form

- Gaining concurrence on final market image (color, shape, size, and package for tablets) prior to formal stability batches or develop a bridging plan (i.e., color change)

- Early CMC risk assessments to support prioritization of experimental studies

- Evaluation of genotoxic impurities: Impurities, impurity controls, and the establishment of Regulatory Starting Materials (RSMs) are related elements of the drug 
substance manufacturing process. With less time to optimize the drug substance process as compared to a traditional development program, it may be necessary to invest more time in negotiation of impurity specifications and designation of RSMs with the FDA. As appropriate, commitments may then be made to reevaluate these elements after launch when the process can be further optimized

Various CMC/GMP development strategies that might facilitate breakthrough drug development are discussed below. In addition, a table from the European Federation of Pharmaceutical Industries Association (EFPIA) Technical Development and Operations Committee (TDOC) Briefing Paper (5) (Annex 2) is included to provide additional examples of opportunities available that might be considered to accelerate traditional CMC approaches for drug development and manufacturing to ensure early access to patients. These proposed strategies will be supplemented with examples (Annex 1) of actual experiences that companies have had working with FDA to implement some of these approaches for expediting approval of breakthrough drug products.

\section{Process and Formulation Development Considerations}

Expedited clinical development programs for breakthrough therapy products will shorten the time available to optimize phase III and commercial manufacturing processes. This will necessitate prioritization of development efforts on process reliability over yield and cost of goods. As a result, process and formulation optimization may need to be deferred to post-approval; if it can be determined, there is no impact on patient safety or product availability. Some activities that might be considered to speed development activities include the following:

- Launching commercial processes with limited experience, but sufficient data to ensure that the process can reliably produce a drug to meet the expected quality safety and efficacy profile and optimize post-approval

- Using data from development material or the clinical supplies, with adequate comparability data to support material from initial commercial process lots, may be needed

- Consider delaying intermediate hold time studies and instead doing straight through processing and scheduling of intermediates to speed process development

- Lock the phase 1/11 drug product formulation and optimize post-approval to avoid need for bioequivalence studies

- If efficacy is indicated in phase I clinical studies, in oncology patients, sponsors may want to strive for a commercial dosage form to be used in the pivotal phase II clinical program

- For biologic products, optimize cell line development early and carry through phase 111 and commercial production

- For small molecule products, the focus should be on the active pharmaceutical ingredient (API) and excipient attributes impacting formulation and DP manufacturability and performance

- Consider close alignment on linkages in control strategies (e.g., particle size distribution impact on dissolution for small molecule drugs) and overarching themes that might apply to both biologics and small molecule drugs (e.g., moisture sensitive API)
Manufacturing Scale and Launch Site Considerations

- Determine, as soon as possible, launch sites for DS and DP, clinical versus commercial

- Clinical manufacturing facilities, used for launch, would need to meet the same quality/GMP expectations as commercial manufacturing facilities

- Key differences for consideration may be:

- Cleaning verification versus cleaning validation

- Multi-product manufacturing, including investigational compounds with limited safety data

- Considering dedicated product contact equipment and/or use of disposables to minimize concerns may be useful. Disposables may also assist with cleaning validation issues

- Gaining concurrence on comparability strategy/protocol for post-approval site changes in advance may lend confidence to manufacturer's ability to ensure sustained supply postlaunch, particularly when expediting launch upon initial approval

- If using a contract manufacturing organization (CMO) for $\mathrm{DS} / \mathrm{DP}$, ensuring there is capacity to allow rapid scale up and to support commercial volumes will be critical

- Consider decoupling drug substance and drug product qualification lots (e.g., using clinical DS for DP qualification), when feasible to save time on the critical path to licensure

- Pivotal clinical studies could be performed with material from different scale and/or site than is intended for long term commercial production (e.g., studies originally expected to be phase II studies could be used as pivotal studies)

- Scaling-up phase III clinical lots to commercial scale for launch with bridging comparability study

\section{Process Validation Considerations}

Process characterization/process validation (PC/PV) studies impacting patient safety must be complete prior to filing. In addition, sufficient process characterization data from clinical and pilot scale lots should be completed to assure process capability and reliability for providing commercial product supply at launch until further PC/ PV activities are completed. The following approaches could be considered for discussion and agreement with FDA.

- Due to the likelihood of having limited manufacturing experience at commercial scale, the number of full-scale validation lots at the time of filing may be lower than a typical application

- Determining if clinical DS could be used for DP process validation, through early alignment with FDA on starting materials (e.g., small molecule products) is critical

- Leveraging process and product platform knowledge (e.g., for monoclonal antibodies) with appropriate justification to speed development

- Leveraging life cycle validation principles, "continued verification"

- Using development experience/smaller scale batches in Process Performance Qualification (PPQ) strategy

- Identifying whether some PC/PV studies could be 
deferred, such as process linkage studies or chromatographic resin reuse at full lifetime

- Considering concurrent validation approaches, based on the FDA Compliance Policy Guide, CPG Section 490.100 (6), for orphan drugs to allow for product distribution concurrent with release of each conformance batch (e.g., batch specific release option). This could enable launch from a commercial site with limited number of batches but is dependent on manufacturer ensuring trust:

- Prior demonstration of manufacturing consistency for clinical process material

- A validation protocol for commercial material and at least one executed batch record at time of filing

- Robust Quality Systems able to effectively manage Corrective and Preventive Actions (CAPAs) and change management

\section{Analytical Development Considerations}

Analytical method development strategies for frontloading of analytical understanding to balance more limited process robustness and support future comparability exercises may include

- A focus on high priority assays, including but not limited to potency for biologics and content, impurities, and dissolution for small molecules to ensure suitability for control system

- Involving commercial quality control (QC) in assay design during development and co-validating, if possible

- Using qualified rather than fully validated methods for internal release and stability testing of qualification lots and completing validation before commercial release

- This approach presents a business risk, if problems arise in validating a method, and should be accompanied with a backup plan requiring retesting lots and/or implementing alternative methods

- Launching from a clinical site with clinical QC release and transferring to commercial site post-launch

\section{Control Strategy Considerations}

Control strategy, based on limited manufacturing experience, but ensuring patient safety and efficacy, may consider,

- Launching with a provisional control system that ensures consistent product and upgrading the control system postapproval with more manufacturing experience and completion of process validation, such as

- Filing with an expanded monitoring program with more tests initially, more assay controls, and justify elimination of some tests post-approval as more knowledge is accumulated

- Filing with broader in process controls (IPC) and product specification acceptance criteria at launch and reevaluating post-approval for specifications that are linked to process consistency

- Filing with preliminary critical process parameters (CPPs) and CQAs
- For small molecules, considering all available data, including (1) dissolution profiles and other critical analytical results, i.e., impurities, solubility, disintegration, etc. during development, (2) ensuring stability specifications are justifiable, if requested by the FDA, and (3) considering sunset specifications for some parameters (e.g., polymorphism)

- Utilizing enhanced modeling techniques, where possible to support conclusions

- Managing second-generation processes through a life cycle approach in the post-approval lifecycle management plan (PALM), which may contain a network of comparability protocols to facilitate life cycle improvements to the product and process

- For critical aspects, consider submitting draft P.2 section (gaps in data sets) for early FDA review and concurrence

\section{Stability Data Considerations}

Accelerated development timelines may limit availability of real-time stability data, thus launching with reduced realtime stability for commercial material may require

- Leveraging stability from early development and clinical batches when formulation remains unchanged and product comparability is demonstrated

- Using forced degradation and stress studies to provide additional supporting and comparability data

- Providing the stability protocol for commercial material

- Gaining FDA concurrence and committing to provide more real-time confirmatory data during review and postapproval

- Enhancing temperature monitoring and control of the product during shipment may be considered until shipping validation studies have been completed

\section{Pharmaceutical Quality System (PQS) Alignment with BT Product Development Considerations}

PQS requirements must be adhered to for breakthrough product development while providing appropriate flexibility to accommodate accelerated activities for breakthrough product development timelines. Thus, the accelerated development PQS strategy for each product will be unique, as it depends on the timing of the BT designation,

- Flexibility, based on molecule, available product, and platform knowledge will be required

- Only those activities with no impact on patient safety or product supply should be considered to be deferred

- A quality risk assessment must be applied to all activities that will be deferred, and the rationale, and controls needed to ensure deferred activities are completed documented

- Some activities that are normally completed prior to license application may need to be deferred and submitted:

- Post-submission, complete at inspection

- Post-inspection, prior to approval

- Post-market commitments

- The manufacturing readiness plan can be used for developing internal filing and inspection readiness checklists to ensure all deferred activities are completed or addressed 
- Any PQS deferrals must be documented in a manufacturing readiness plan and monitored to ensure completion

\section{BALANCING RISK OF LESS CMC DATA AT TIME OF FILING VERSUS PATIENT BENEFIT}

In spite of front-loading certain critical product and process characterization activities, it may not be possible in the limited timeframes available to complete all CMC/GMP activities at the time of filing and launch of a breakthrough product. To address this possibility, manufacturers should develop a manufacturing readiness plan, which aligns the timeline for completing the manufacturing activities with those of the clinical development program. This plan should address all manufacturing sites and their suitability and readiness for development and launch of the breakthrough product, the design and implementation of critical characterization tools, the validation approach for process and methods, stability data to support adequate expiration dating for the product, and delineation of responsibilities for the development and commercial teams in addressing these issues. Where gaps exist in completing certain activities, a risk assessment should be performed, addressing the availability of less CMC information at the time of filing and product launch versus patient benefit. This should be coupled with a risk mitigation plan to address these risks either prior to launch or through the use of a post-approval life cycle management plan.

The manufacturing readiness plan and risk assessment should form the basis for discussion and agreement with FDA prior to filing the marketing application. As part of this plan, below are several proposed examples of CMC/GMP activities that may be considered as incomplete at the time of filing and launch of a breakthrough drug product:

- Process validation with fewer than the standard number of full-scale manufacturing runs

- Process characterization, e.g., long duration elements like resin reuse, validation of intermediate process hold times, or extending limit of in vitro cell age for life cycle management of a biologic product

- Available real-time stability data on commercial product

- Validated transfer to commercial manufacturing site/scale, though some level of assurance will still be necessary regarding transfer for biologics

- Provisional control system that ensures consistent product with need to upgrade post-approval

- Reliable process capable of meeting initial product demand with need to optimize process yield and performance postapproval

- Phase I/II formulation for launch with potential need to optimize post-approval

A fundamental assumption is that risk assessments demonstrate that having less data at the time of filing and launch of a breakthrough product will not compromise patient safety or product supply. Completion of any deferred CMC activities should be documented in a comprehensive PALM that is part of the marketing application and contains detailed timelines, deliverables, and types of regulatory filing to be completed post-approval.

\section{FLEXIBILITY IN TYPE AND EXTENT OF MANUFACTURING DATA FOR MARKETING APPROVAL OF BREAKTHROUGH DRUG}

FDA approval standards for marketed drugs require demonstration of substantial evidence of effectiveness, safety, and product quality. FDA's expectation for pharmaceutical quality is the same for all drugs. However, FDA regulations for orphan drugs do allow for flexibility and scientific judgment in applying approval standards, in terms of the amount and type of data needed for a particular drug to meet the statutory standards. This rationale is stated in FDA's final guidance on Expedited Programs for Serious Diseases (2) which states that the "FDA may exercise some flexibility on the type and extent of manufacturing information that is expected at the time of submission and approval for certain components (e.g., stability updates, validation strategies, inspection planning, manufacturing scale-up)." Open and transparent discussions with FDA on balancing (and mitigating) risk of less CMC/GMP information at the time of filing versus patient benefit should take place prior to filing the marketing application.

\section{SPONSOR/FDA INTERACTIONS DURING DEVELOPMENT AND REVIEW OF BREAKTHROUGH DRUGS}

In addition to a risk-based, front-loaded development plan undertaken by the manufacturer to expedite ratelimiting steps in $\mathrm{CMC} / \mathrm{GMP}$ for breakthrough drug products, the agency can work with manufacturers on risk-based solutions that facilitate expedited development and review timelines without compromising availability of an adequate supply of safe and effective products for patients. A few areas for consideration are as follows:

- The traditional and time-consuming process of formal meeting requests, scheduling, briefing documents, and written responses may not be appropriate in the environment of an accelerated breakthrough therapy drug development program. More flexible approaches to ensuring information exchange and understanding should be considered to facilitate expediting development and review. Formal meetings should be reserved for more comprehensive program discussions or critical review milestones.

- Soon after receiving a breakthrough designation, manufacturers should work with FDA on a plan for early and active engagement to schedule and conduct meetings during development to reach agreement on best path forward

- Consider designating a CMC/GMP point of contact, within both sponsor and FDA, to triage meeting requests and sponsor questions

- Set up secure email to facilitate information exchange

- Agree upon schedule of important review milestones and turnaround timeframes for information requests

- Discuss use of "negotiated amendment" approach to submit agreed upon data packages during the review, for example: 
- Submission of the dissolution method development report and dissolution specification setting strategy for early review by FDA Biopharmaceutics reviewers

- Additional real-time stability data on commercial product

- Additional batch data to support validation

- Discuss rolling submission of module 3 components to enable more rapid access to $\mathrm{CMC}$ and facility data to facilitate preapproval inspection scheduling and conduct; Gain early and frequent access to reviewers via teleconferences to resolve questions, avoid delays, and provide clarity on specific concerns

- For small molecules, flexibility on the qualification of regulatory starting materials (RSMs), impurities, and impurity controls, perhaps accepting something on an interim basis with a post-marketing commitment to reevaluate these controls after launch. Impurities and their associated controls, including RSMs, should be considered in light of the clinical indication and the potentially life-saving nature of the drug. It may be necessary for drugs which have not been fully optimized at the time of launch to allow for wider initial controls which can be adjusted and refined as more experience is gained in commercial manufacturing provided product safety and quality will not be impacted

\section{CONCLUSION}

Breakthrough therapies offer significant patient benefits, but the reduced timelines introduce significant $\mathrm{CMC} / \mathrm{GMP}$ challenges for product development as well as resource commitments to align the development and commercial organizations. Each breakthrough drug development program will have different risks and constraints, so the specific $\mathrm{CMC} /$ GMP approaches will vary by product and timing of the breakthrough designation. Through careful planning and a thorough understanding, by all parties, of the requirements and timeframes, some activities may be optimized post-approval. Leveraging prior knowledge, platform data, and use of comparability protocols are key considerations for developing a breakthrough drug product. Additional considerations include the use of initial product supply from a clinical process or site, use of supportive stability data from representative pilot scale lots, delaying certain process validation requirements not directly related to patient safety, and consideration of broader product quality acceptance ranges for non-critical quality attributes until further manufacturing experience is gained. As a result, these programs will generate significant post-approval CMC efforts and phase IV commitments to address control system updates, process optimization where needed, and site transfers. The key to success is open and transparent communications with FDA to ensure the development program delivers an adequate supply of safe and efficacious product to patients.

\section{ACKNOWLEDGMENTS}

This article was developed as an issue brief for the Friends of Cancer Research Public Forum on Manufacturing Readiness, held on June 10, 2015. The authors would like to thank the participants of the public meeting and, above all, the

panelists who facilitated the discussions: Earl Dye, Annie Sturgess, Kimberly May, Usha Ramesh, Keith Cockerill, John Groskoph, Emanuela Lacana, Paul Seligman, Diane Zezza, Tom O'Connor, Tim Watson, Christopher Joneckis, and Sarah Pope Miksinski. We also thank staff at Friends of Cancer Research, in particular Marina Kozak, for their expertise, thoughtful input on the manuscript, and logistical support.

\section{COMPLIANCE WITH ETHICAL STANDARDS}

Author contributions

the manuscript.

\section{ANNEX 1}

Case studies of actual challenges encountered by sponsors during BT development and flexibilities that were agreed upon with the FDA to ensure product safety and availability at the time of approval.

\section{Biologics}

\section{Example no. 1:}

Genentech/Roche-Gazyva ${ }^{\circledR}$ (obinutuzumab) is a humanized monoclonal antibody approved for the treatment of lymphoma. Acting as an immunomodulator, it targets CD20, killing B cells. Gazyva was the first FDA-designated breakthrough therapy approved by the U.S. FDA in November of 2013. Breakthrough therapy designation was granted for Gazyva late in the development cycle, just prior to the Biologics License Application (BLA) filing. Because of the late stage of designation as a Breakthrough Therapy, most CMC development activities for Gazyva had been completed

- However, to allow for earlier launch, the FDA encouraged conversion of phase III clinical material to launch material in order to accommodate an early launch ( 1 month sooner)

- Detailed assessments of clinical material took place during PDUFA V mid-cycle and late-cycle meetings with FDA and during PAI at the DS manufacturing site

- Same commercial manufacturing facilities and same scale of manufacture

- Same manufacturing processes planned (very minor changes)

- Transition from clinical to commercial CoA (met all commercial specifications)

- Qualified persons requested written endorsement from FDA to release

- Very supportive interaction with FDA regarding conversion of clinical material to commercial launch material to get this medicine to chronic lymphocytic leukemia (CLL) patients quickly

\section{Example no. 2:}

Merck \& Co.-Keytruda ${ }^{\circledR}$ (pembrolizumab) is the first PD-1 blocking drug approved by the U.S. FDA, in September of 2014, for the treatment of patients with advanced or unresectable melanoma who are no longer responding to other drugs. At 
the time, breakthrough therapy status was granted to Keytruda®, clinical supplies were only manufactured on a small clinical scale, clinical development was in phase I, and CMC development was stage appropriate, in early stages

- Expediting CMC readiness to meet clinical timelines meant decoupling DS Process Performance Qualification (PPQ) from DP PPQ, enabling almost parallel execution and completion of DS and DP PPQ activities, both of which were rate-limiting to the $\mathrm{CMC}$ file. This was enabled by ensuring no significant process changes were implemented between the clinical GMP DS batches used for DP PPQ and the subsequent DS PPQ batches, saving 4-6 months in the development timeline without incurring additional quality or patient safety/efficacy risk

- To meet the projected commercial and clinical demand, an additional drug substance manufacturing site was rapidly brought online prior to BLA filing. Through multiple interactions with the FDA, licensure was sought for two drug substance manufacturing facilities, one that was the initial clinical supply site and, a second larger CMO site (licensure of this site was based on a strong analytical comparability package, the approach and content of which were discussed with the FDA via frequent interactions)

- The FDA partnership was critical to rapid resolution of multiple CMC issues, especially since this was Merck's first monoclonal antibody filing with the FDA. During the final stages of the review of the BLA application, the field office site inspections were not synchronized with early action by the review division-this resulted in removal of one of the manufacturing sites from the BLA, which was subsequently submitted for review and approved very rapidly

- In addition to the rapid pace of development of this molecule, along with multiple sites, the dosage form also transitioned from a lyophilized powder for solution for infusion to a liquid vial. This supply strategy was discussed and reviewed with FDA, in advance, resulting in the recent approval of the post-approval supplement for the liquid vial, based on analytical comparability in the previously agreed upon strategy

- A process/product-specific host cell protein (HCP) method for measurement of host cell impurities in the drug substance was not in place at the time of designation. Upon FDA review, a well-characterized commercially available HCP assay, demonstrating appropriate coverage and clearance in the process, was used for initial commercial release. During BLA review, a postmarketing commitment to develop a process/productspecific HCP assay was agreed to. This allowed development, bridging, and validation of this HCP method off critical path to initial approval, ensuring that the interim solution did not pose any patient safety/efficacy risk. Alternatively, inclusion of the process/product-specific $\mathrm{HCP}$ assay in the BLA filing would have resulted in a minimum of 6-9-month delay

- The importance of frequent and data-driven interactions with the FDA was critical to the success of CMC development for this drug

\section{Example no. 3:}

Bristol-Myers Squibb-Opdivo® (nivolumab) was approved in December, 2014. Opdivo works by inhibiting the PD-1 protein and is intended for patients who have been previously treated with ipilimumab, for melanoma patients whose tumors express BRAF V600, and for use after treatment with ipilimumab and a BRAF inhibitor. The following flexibilities allowed for development of a complete package:

- Final cell-based bioassay was not available until after PPQ batches

- Used frozen samples (release and stability) to allow testing following method validation to justify acceptance criteria

- DS process changes allowed for improved robustness and facilitated future transfers to additional sites

- Introduced modifications to downstream or purification processing steps prior to manufacture of commercial supplies; no change in cell line or upstream process

- Type B and type C meeting to align on strategy; Provided preliminary comparability data, including

Comparison of release and extended characterization analytical data

Side-by-side degradation profile at stress conditions

Full scale in-process control data comparison

- Able to bridge stability data to allow expiry to be based on studies performed using material from the clinical process

- Endotoxin

- Low endotoxin recovery observed with original (kinetic) method used for drug substance

- Type B meeting to align on proposed strategy

- Changed to gel clot method during BLA review

- Addition of $40 \mathrm{mg} / \mathrm{vial}$ presentation with limited formal stability data

- Same formulation and glass vial as used for $100 \mathrm{mg} / \mathrm{vial}$ presentation

- Type $\mathrm{C}$ meeting to align on stability strategy to support proposed expiry

\section{Example no. 4:}

Amgen-Blincyto ${ }^{\circledR}$ (blinatumomab) was approved in December, 2014, to treat patients with Philadelphia chromosome-negative precursor B cell acute lymphoblastic leukemia (B cell ALL). It is a first-in-class bispecific $\mathrm{T}$ cell engager (BiTE®) antibody construct that binds CD19 on B cells and CD3 on T cells, inducing a cytotoxic $\mathrm{T}$ cell response to kill target $\mathrm{B}$ cells. Blincyto received BT designation 2.5 months prior to BLA submission, and accelerated approval (11-week BLA review) was based on phase 2 data for relapsed or refractory B cell ALL

- With a history of multiple sponsors and manufacturing sites and six manufacturing processes, there was

- No clinical experience with the commercial manufacturing process and limited process experience due to complex manufacturing history

- FDA requested several months acceleration of drug substance contract manufacturing to enable early inspection and faster review

- A dissolution issue with raw material delayed initiation of drug substance manufacturing from the date agreed upon with FDA

- The FDA agreed to inspect earlier process steps and required a commitment to provide results of raw material investigation and product quality data when available as 
well as evidence of existing inventory to supply the market

- The discontinuity in process characterization was addressed by extensive FDA information requests to understand process robustness

- Post-marketing commitments to qualify tests for certain inprocess sample types and to complete drug substance and drug product container closure leachate studies allowed for timely submission and approval

\section{Small Molecules}

\section{Example no. 5:}

Pfizer-Ibrance ${ }^{\circledR}$ (palbociclib) was granted accelerated approval, by the US FDA in early 2015, to treat postmenopausal women with estrogen receptor (ER)-positive, human epidermal growth factor receptor 2 (HER2)-negative metastatic breast cancer by inhibiting cyclindependent kinases (CDKs) 4 and 6. During commercial scale-up, the manufacturer identified a drop in dissolution performance at the end of each batch. This phenomenon did not occur at smaller manufacturing scale of the drug

- In order to continue uninterrupted supply to the clinical study while this issue was being investigated, a batch cutoff at $85 \%$ was instituted by the sponsor to throw away the final $15 \%$ of each batch

- The FDA was informed of the issue and agreement was obtained that the $85 \%$ cutoff was an appropriate interim measure until a permanent corrective action could be identified

- The applicant identified a set of successful modifications to the encapsulator hopper to improve powder flow and eliminate over-lubrication of the tail end of the batch. Stratified data across multiple batches and strengths confirmed the corrective action was successful

- Ultimately, the $85 \%$ cutoff was successfully phased out for the commercial process and all future clinical batches

Example no. 6:

Pharmacyclics-Imbruvica ${ }^{\circledR}$ (ibrutinib), a first in class, selective, small molecule inhibitor of Bruton's tyrosine kinase (BTK), was granted breakthrough therapy status for three indications in early 2013. Imbruvica ${ }^{\circledR}$ received its first approval under breakthrough therapy designation (BTD) by the FDA on November 13, 2013, for the treatment of patients with mantle cell lymphoma who have received at least one prior therapy. Subsequently, Imbruvica ${ }^{\circledR}$ was approved by the FDA for three additional indications: the treatment of patients with chronic lymphocytic leukemia who have received at least one prior therapy, chronic lymphocytic leukemia patients with $17 \mathrm{p}$ deletion (under BTD), and for the treatment of patients with Waldenstrom's macroglobulinemia (under BTD). Because BTD was granted at the end of phase 2 clinical studies, development timelines for Imbruvica were shortened by about 12-18 months. CMC development activities were on the critical path for the NDA submission and commercial launch.

- One of the regulatory starting materials Pharmacyclics proposed was not accepted by the FDA. FDA requested that the regulatory starting material should be separated from the DS by additional synthetic steps. The material which was custom manufactured for Pharmacyclics was therefore designated as an intermediate and the manufacturing process of this intermediate was added to the commercial manufacturing process. The site was rapidly readied for pre-approval inspections

- At the CMC-specific pre-NDA meeting, several key issues were discussed with the FDA and agreements obtained to expedite commercial readiness. Agreement was obtained on regulatory starting materials, impurity qualification strategy, validation strategy, etc.

- To meet the compressed timelines for NDA submission and approval, PPQ activities of DP and PPQ of DS were conducted in parallel. This was made possible because no major process changes were implemented between DS manufacturing process used to manufacture the pivotal clinical batches and eventual commercial process

- The commercial DS manufacturing site was different from the site where earlier clinical batches were manufactured. Comparability data of clinical batches to commercial batches was used to support the change. Both clinical process and commercial process used similar control strategy and no major changes to the manufacturing process were made between the two sites

- An alternate more discriminating dissolution method was developed and validated prior to NDA submission. However, available data generated using the new method was limited and not sufficient to propose a specification using this method. A commitment was made to collect additional data using the new method and revise the dissolution specification post-approval

- Responses to FDA queries and request for information during review were completed promptly with turnaround time of 24-48 h

- Labels, cartons, and other launch materials were printed at risk in order to minimize delay in commercial launch after approval

\section{ANNEX 2 (5)}

Illustrative Examples of Adaptations of Traditional CMC Development and Manufacturing Approaches for APIs and Drug Products to Ensure Early Access

The following table from the European Federation of Pharmaceutical Industries Association (EFPIA) Technical Development and Operations Committee (TDOC) Briefing Paper (Annex 2) illustrates some expedited approaches which a company may take (MAPPs aligned approach) to ensure early access of medicines for the 
Medicines Adaptive Pathways to Patients Initiative (MAPPs) in Europe. Note this table is not intended to be comprehensive and is for illustrative purposes only. Most aspects of the proposals are valid for small molecules/new chemical entities (NCEs) as well as large molecules/biotech products.

$\begin{array}{ll}\text { Topic } & \text { Traditional approach } \\ \text { Formulation } & \text { Commercial formulation developed and } \\ & \text { optimized; comparability to pivotal clinical } \\ & \text { formulation demonstrated in dossier }\end{array}$

Packaging

Analytical methods

Specification

Impurity assessment

Shelf-life

Process development

Process validation

Scale of production
Optimized, based on minimum requirements for protection

Developed and validated

Established and documented Supported by extensive dataset

Impurities identified, risk assessed and controlled

Controlled mainly by process knowledge rather than specification testing

Shelf-life at launch based upon defined length of stability data on defined batch types/sizes (ICH Q1A)

Post-approval extension as further data emerges

Complete package at filing

Process supported by extensive development studies

Prospective or continued process verification

Commercial scale

\section{MAPPs aligned approach}

Use of clinical formulation or limited optimisation of selected market form

Where relevant, comparability of launch formulation to pivotal clinical formulation demonstrated in dossier

Where relevant/known, planned commercial formulation described and a PACM Protocol to demonstrate comparability to pivotal clinical formulation in the dossier

Potential for use of "maximum protection pack" to mitigate limited shelf-life

Developed and validated

Established and documented; possibly broader specifications as little data are available

May include more elements than traditional specification due to limited data set and/or some parameters where the data will be reported but acceptance criteria not defined

Commitment to update (rationalize) after $\mathrm{x}$ time or $\mathrm{y}$ batches, based on pre-defined criteria and to reassess the control strategy.

Impurities identified, risk assessed and controlled

Higher level of control by specification testing (could include intermediates) may be needed until sufficient data available to support greater reliance on process control

Launch product will be supported by (ongoing) stability studies, but ICH-conform data may be limited. Negotiate employment of lean stability strategies (including stress conditions), use of stability models, and extrapolation for supporting shelf-life with competent authorities, enhanced use of scientifically relevant supporting data from earlier batches, and possibly more than one batch annually in ongoing stability

Support of adequate shelf-life with use of highly protective packaging/restrictive storage conditions as appropriate to the elicited degradation mechanisms

Post-approval strategies will depend on formulation strategy and may also involve novel approaches

Partly based on platform knowledge, to be refined as more batches/materials are investigated

May be based on proven acceptable ranges (or set points) until data set complete; more reliance on end testing for product release

Seek regulators' agreement to a concurrent validation approach, including extended monitoring

Small commercial scale

Scale-up protocol defined 
Sites of production

Viral clearance validation

Inspection of facility

Cleaning method

Cleaning validation

DMFs (where used)
Commercial manufacturing site Existing cGMP clearance or inspection-ready Multiple sites may be included

Validated in small scale

GMP certificate available for commercial use of the facility

Established

Validated

Submitted in close conjunction with MAA
May be clinical manufacturing site

Existing cGMP clearance (possibly only MIA-IMP)

Inspection-ready; product history available to support approval of clinical site for commercial launch

Site addition PACM Protocol defined

If appropriate platform data are available: include such data in dossier, validate in small scale prior to launch, and agree mechanism for provision of data to competent authorities

Acceptance of GMP certificate for IMP manufacture or, where facilities are outside the EU, the acceptance of QP Declaration for imported API/product

Established

Appropriate analyses on batch-wise basis and and/or concurrent validation

Negotiate early submission/pre-assessment to mitigate risk of landing on critical review path

\section{ANNEX 3: ABBREVIATIONS}

$\begin{array}{ll}\text { API } & \text { active pharmaceutical ingredient } \\ \text { BT } & \begin{array}{l}\text { breakthrough therapy } \\ \text { corrective and preventative actions } \\ \text { CAPA }\end{array} \\ \text { CMC } & \begin{array}{l}\text { chemistry, manufacturing, and control } \\ \text { critical process parameters }\end{array} \\ \text { CPP } & \begin{array}{l}\text { critical quality attributes } \\ \text { DP }\end{array} \\ \text { drug product } \\ \text { GMP } & \text { good manufacturing practices } \\ \text { IPC } & \text { in-process control } \\ \text { MAPPs } & \text { Medicines Adaptive Pathways to Patients Initiative } \\ \text { PAI } & \text { pre-approval inspection } \\ \text { PALM } & \text { post-approval life cycle management plan } \\ \text { PC/PV } & \text { process characterization/process validation } \\ \text { PC/PV } & \text { process characterization/process validation } \\ \text { PK } & \text { pharmacokinetic } \\ \text { PPQ } & \text { process performance qualification } \\ \text { PQS } & \text { pharmaceutical quality systems } \\ \text { PQS } & \text { pharmaceutical quality systems } \\ \text { RSM } & \text { regulatory starting materials } \\ \text { QC } & \text { quality control } \\ \text { QTTP } & \text { quality target product profile }\end{array}$

\section{REFERENCES}

1. Pub. L. No. 112-144, $\S 902,126$ Stat. 993. Section 506(a) of the Federal Food Drug and Cosmetic (FD\&C) Act, as added by section 902 of Food and Drug Administration Safety and Innovation Act (FDASIA). 2012.

2. Sawyers C. et al. Developing standards for breakthrough therapy designation. Issue Brief from the Conference on Clinical Cancer Research. 2012. http://www.focr.org/sites/default/files/CCCR12Breakthrough.pdf

3. U.S. Department of Health and Human Services, Food and Drug Administration, Center for Drug Evaluation and Research (CDER) Center for Biologics Evaluation and Research (CBER). 2014. Guidance for industry: expedited programs for serious conditions-drugs and biologics. 21 CFR part 312

4. Friends of Cancer Research Public Forum on Manufacturing Readiness. 2015. http://www.focr.org/events/examining-manufacturingreadiness-breakthrough-drug-development-0

5. European Federation of Pharmaceutical Industries and Associations (EFPIA) Technical Development and Operations Committee (TDOC). EFPIA TDOC briefing paper on Medicines Adaptive Pathways to Patients Initiative (MAPPs) - CMC challenges and opportunities

6. U.S. Department of Health and Human Services, Food and Drug Administration. Process validation requirements for drug products and active pharmaceutical ingredients subject to pre-market approval. 2004. http://www.fda.gov/ICECI/ComplianceManuals/ CompliancePolicyGuidanceManual/ucm074411.htm 